\title{
Solvability of Linear Functional Equations in Lebesgue Spaces
}

By

\author{
Sen-Yen SHAW*
}

\section{§1. Introduction}

Let $A$ be a closed linear operator on a Banach space $X$. This paper is concerned with the solvability and approximate solutions of the equation $A x=y$ for a given $y \in X$, especially when $X$ is a Lebesgue space $L_{p}, 1 \leqq p<\infty$. The domain, null space, and range will be denoted by $D(A), N(A)$, and $R(A)$, respectively.

Let $\left\{A_{\alpha}\right\}$ and $\left\{B_{\alpha}\right\}$ be two nets, indexed by a directed set $\mathcal{A}$ of bounded linear operators on $X$ with the following properties:

(a) $\left\|A_{\alpha}\right\| \leqq M$ for all $\alpha \in \mathcal{A}$;

(b) $R\left(B_{\alpha}\right) \subset D(A)$ and $B_{\alpha} A \subset A B_{\alpha}=I-A_{\alpha}$ for all $\alpha \in \mathcal{A}$;

(c) $R\left(A_{\alpha}\right) \subset D(A)$ for all $\alpha \in \mathcal{A}, \mathrm{w}-\lim _{\alpha} A A_{\alpha} x=0$ for all $x \in X$, and $\mathrm{s}$ $\lim _{\alpha} A_{\alpha} A x=0$ for all $x \in D(A)$.

(d) $B_{\alpha}^{*} x^{*}=\phi(\alpha) x^{*}$ for all $x^{*} \in R(A)^{\perp}\left(=N\left(A^{*}\right)\right.$ in case $\left.D(A)=X\right)$ with $\lim _{\alpha}|\phi(\alpha)|=\infty$.

We call $\left\{A_{\alpha}\right\}$ a system of almost invariant integrals for $A+I$ and $\left\{B_{\alpha}\right\}$ the system of companion integrals. The terminologies go back to those of Eberlein [4] and Dotson [2] for the case $A=T-I$ with $T$ bounded. The following two theorems concerning the convergence of $\left\{A_{\alpha} x\right\}$ and $\left\{B_{\alpha} y\right\}$ have been established in $[8]$ :

(i) $\left\{A_{\alpha} x\right\}$ converges if and only if it contains a weakly convergent subnet, if and only if $x \in N(A) \oplus \overline{R(A)}$, and the mapping $P: x \rightarrow \mathrm{s}-\lim _{\alpha} A_{\alpha} x$ is a bounded projection with $R(P)=N(A), N(P)=\overline{R(A)}$ and $D(P)=N(A) \oplus \overline{R(A)}$,

(ii) $\left\{B_{\alpha} y\right\}$ converges if and only if it contains a weakly convergent subnet,

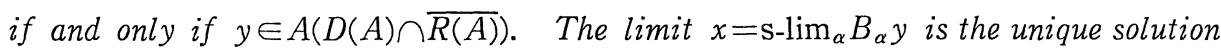
of the equation $A x=y$ in $\overline{R(A)}$.

In a reflexive space, the weak sequential precompactness of bounded sets

Communicated by S. Matsuura, January 5, 1990.

* Department of Mathematics, National Central University, Chung-Li, Taiwan, R. O.C. 
implies that $D(P)=X$ and $R(A)=A(D(A) \cap \overline{R(A)})$. The following theorem [8, Corollary 1.8] is then easily deduced form (ii).

Theorem 1. If $X$ is a reflexive space, then, under the conditions (a), (b), (c), and (d), the following statements are equivalent:

(1) $y \subseteq R(A)$;

(2) $\left\{B_{\alpha} y\right\}$ is bounded;

(3) There is a subnet $\left\{B_{\beta}\right\}$ of $\left\{B_{a}\right\}$ such that $x=\mathrm{w}-\lim _{\beta} B_{\beta} y$ exists;

(4) $x=\mathrm{s}-\lim _{\alpha} B_{\alpha} y$ exists.

Moreover, the $x$ in (3) and (4) is the unique solution of $A x=y$ in $\overline{R(A)}$.

This theorem holds in particular for any Lebesgue space $L_{p}(S, \Sigma, \mu)$ with $1<p<\infty$. In general, while the implications " $(3) \Leftrightarrow(4) \Rightarrow(1) \Rightarrow(2)$ " always hold (due to (ii), (a), and (b)), the other two implications " $(2) \Rightarrow(1)$ " and "(1) $\Rightarrow(4)$ " may not hold in a nonreflexive space (cf. [9] and [8, Remark 1.7]). However, with some additional assumption, we shall prove in section 2 the following positive result for $L_{1}(S, \Sigma, \mu)$.

Theorem 2. Let $X=L_{1}(S, \Sigma, \mu)$ wih $\mu$ a $\sigma$-finite measure. If $\left\{A_{\alpha}\right\}$ and $\left\{B_{a}\right\}$ satisfy (a) with $M=1,(\mathrm{~b}),(\mathrm{c})$, and (d), then (1) and (2) are equivalent. If, in addition to the above assumption, $\mu$ is a finite measure and $\left\|A_{\alpha} f\right\|_{\infty} \leqq K\|f\|_{\infty}$ for all $f \subseteq L_{\infty}(S, \Sigma, \mu)$ and $\alpha \subseteq \mathcal{A}$, then the statements (1), (2), (3), and (4) are equivalent, and the limit $x$ in (3) and (4) is the unique solution of $A x=y$ in $\overline{R(A)}$.

These general theorems can be used to study the solvability and various approximate solutions of the linear functional equation $A g=f$ in $L_{p}(S, \Sigma, \mu)$, $1 \leqq p<\infty$. For illustration we shall display in sections 3 and 4 applications to $n$-times integrated semigroups and cosine operator functions, respectively. Applications to other methods of solving $(I-T) x=y$ such as those considered in [8] are also possible. In particular, theorems of Lin and Sine [7], and of Krengel and Lin [6, Theorem 3.1] can be deduced from this result. In section 3 , the almost everywhere pointwise convergence of the approximate solutions of $A g=f$ will also be observed for the case that $A$ is the generator of a $C_{0}$ semigroup of contractions on $L_{1}(S, \Sigma, \mu)$ that also fulfills the condition that $\|T(t) f\|_{\infty} \leqq K\|f\|_{\infty}$ for all $f \in L_{1} \cap L_{\infty}$ and $t \geqq 0$.

\section{§2. Proof of Theorem 2}

Suppose (1) holds, i.e., $y=A x$. Then (a) and (b) imply that $\left\|B_{\alpha} y\right\|=$ $\left\|B_{a} A x\right\|=\left\|\left(I-A_{\alpha}\right) x\right\| \leqq(1+M)\|x\|$ for all $\alpha \in \mathcal{A}$, i. e., (2) holds.

Conversely, if $\left\{B_{\alpha} y\right\}$ is bounded, we first show that $A_{\alpha} y \rightarrow 0$. Indeed, (d) implies that for each $x^{*} \in R(A)^{\perp}$ we have 


$$
\left\|B_{\alpha} y\right\|\left\|x^{*}\right\| \geqq\left|\left\langle B_{\alpha} y, x^{*}\right\rangle\right|=\left|\left\langle y, \phi(\alpha) x^{*}\right\rangle\right|=|\phi(\alpha)|\left|\left\langle y, x^{*}\right\rangle\right|,
$$

which would be unbounded unless $\langle y, x\rangle=0$. Hence $y$ belongs to ${ }^{\perp}\left(R(A)^{\perp}\right)=$ $\overline{R(A)}$. This fact with assumptions (a) and (c) implies that $A_{\alpha} y$ converges in norm to 0 .

Next, let $\operatorname{LIM}_{\beta}$ be a Banach limit on the space of bounded functions on $\mathcal{A}$, and define a linear functional $q$ on $L_{1}(\mu)^{*}=L_{\infty}(\mu)$ by $q\left(x^{*}\right)=\operatorname{LIM}_{\beta}\left\langle B_{\beta} y, x^{*}\right\rangle$, $x^{*} \in L_{\infty}(\mu)$. Then $q$ belongs to $X^{* *}=L_{\infty}(\mu)^{*}=b a(S, \Sigma, \mu)$, the space of bounded finitely additive measures (=charges) $\ll \mu$, and $\|q\| \leqq \sup _{\alpha}\left\|B_{\alpha} y\right\|$. We have for $x^{*}=X^{*}$ and $\alpha \in \mathcal{A}$

$$
\begin{aligned}
{\left[A_{\alpha}^{* *} q\right]\left(x^{*}\right)=q\left(A_{\alpha}^{*} x^{*}\right) } & =\operatorname{LIM}_{\beta}\left\langle B_{\beta} y, A_{\alpha}^{*} x^{*}\right\rangle \\
& =\operatorname{LIM}_{\beta}\left\langle\left(I-B_{\alpha} A\right) B_{\beta} y, x^{*}\right\rangle \\
& =\operatorname{LiM}_{\beta}\left\langle\left(B_{\beta} y-B_{\alpha}\left(I-A_{\beta}\right) y, x^{*}\right\rangle\right. \\
& =\operatorname{LIM}_{\beta}\left\langle\left(B_{\beta} y, x^{*}\right\rangle-\left\langle B_{\alpha} y, x^{*}\right\rangle+\lim _{\beta}\left\langle A_{\beta} y, B_{\alpha}^{*} x^{*}\right\rangle\right. \\
& =q\left(x^{*}\right)-\left\langle B_{\alpha} y, x^{*}\right\rangle,
\end{aligned}
$$

(b) and the fact that $P y=0$ having been used. Hence $A_{\alpha}^{* *} q=q-B_{a} y$ for all $\alpha \in \mathcal{A}$.

$L_{1}(S, \Sigma, \mu)$ can be identified, via the Radon-Nikodym theorem, with $M(S, \Sigma, \mu)$, the subspace of $b a(S, \Sigma, \mu)$ which consists of all countably additive measures $\ll \mu$. Decomposing $q=q_{1}+q_{2}$ with $q_{1} \in M(S, \Sigma, \mu)$ and $q_{2}$ a pure charge (cf. [12]), and using the contraction assumption and the fact that the norm of an element of $b a(S, \Sigma, \mu)$ is the sum of the norms of its two parts, we obtain the estimate:

$$
\begin{aligned}
\left\|q_{2}\right\| \geqq\left\|A_{\alpha}^{* *} q_{2}\right\| & =\left\|q_{1}-B_{\alpha} y-A_{\alpha}^{* *} q_{1}+q_{2}\right\| \\
& =\left\|q_{1}-B_{\alpha} y-A_{\alpha} q_{1}\right\|+\left\|q_{2}\right\|,
\end{aligned}
$$

which shows that $q_{1}=B_{\alpha} y+A_{\alpha} q_{1} \in D(A)$ and $A q_{1}=A B_{\alpha} y+A A_{\alpha} q_{1}=y-A_{\alpha} y+$ $A A_{\alpha} q_{1}$ for all $\alpha \in \mathcal{A}$. Taking limits yields that $y=A q_{1} \in R(A)$. Thus we have proved the equivalence of (1) and (2).

Since, as mentioned in the introduction, the conditions (3) and (4) are equivalent to that $y$ belongs to $A(D(A) \cap \overline{R(A)})=A(D(A) \cap D(P))$, which is equal to $R(A)$ when $D(P)=X$, the second part of Theorem 2 follows from the next lemma.

Lemma 3. Let $(S, \Sigma, \mu)$ be a finite measure space and let $\left\{A_{\alpha}\right\}$ and $\left\{B_{\alpha}\right\}$ be bounded operators on $X=L_{1}(S, \Sigma, \mu)$ as well as on $L_{\infty}(S, \Sigma, \mu)$ which satisfy (b), 
(c), and (d). Suppose further that $\left\|A_{\alpha} f\right\|_{1} \leqq M\|f\|_{1}$ for all $f \in L_{1}(S, \Sigma, \mu)$ and $\left\|A_{\alpha} h\right\|_{\infty} \leqq K\|h\|_{\infty}$ for all $h \subseteq L_{\infty}(S, \Sigma, \mu)$ and for all $\alpha \in \mathcal{A}$. Then $\left\{A_{\alpha} f\right\}$ converges in $L_{1}(S, \Sigma, \mu)$ for all $f$ in $L_{1}(S, \Sigma, \mu)$.

Proof. If $h$ is a simple function, then $\left|\int_{E}\left(A_{\alpha} h\right) d \mu\right| \leqq K\|h\|_{\infty} \mu(E)$ which converges to 0 uniformly for $\alpha \in \mathcal{A}$ as $\mu(E) \rightarrow 0$. Hence $\left\{A_{\alpha} h ; \alpha \in \mathcal{A}\right\}$ is weakly sequentially precompact in $L_{1}(S, \Sigma, \mu)$ (see [3, Corollary IV.8.11]). It follows from (i) in the introduction that $\left\{A_{\alpha} h\right\}$ converges in $L_{1}(S, \Sigma, \mu)$. Since the set of all simple functions is dense in $L_{1}(S, \Sigma, \mu)$ and since $\left\{A_{\alpha}\right\}$ is uniformly bounded, the convergence of $\left\{A_{\alpha} f\right\}$ holds for all $f$ in $L_{1}(S, \Sigma, \mu)$.

\section{$\S 3$. Generators of $n$-times Integrated Semigroups}

A strongly continuous family $\{T(t) ; t \geqq 0\}$ of bounded operators on $X$ is called a $n$-times integrated semigroup if $T(0)=I$ and $T(t) T(s)=T(t+s)(t, s \geqq 0)$ in case $n=0$, and if $T(0)=0$ and

$$
T(t) T(s)=\frac{1}{(n-1) !}\left\{\int_{t}^{t+s}(t+s-r)^{n-1} T(r) d r-\int_{0}^{s}(t+s-r)^{n-1} T(r) d r\right\} \quad(t, s \geqq 0)
$$

in case $n \geqq 1$. A 0 -times integrated semigroup is just the classical $C^{0}$-semigroup. $T(\cdot)$ is said to be non-degenerate if $T(t) x=0$ for all $t>0$ implies $x=0$, and exponentially bounded if $\|T(t)\| \leqq M e^{w t}$ for some $M \geqq 1, w>0$ and for all $t \geqq 0$. For a non-degenerate and exponentially bounded $T(\cdot)$ there exists a uniquely determined closed operator $A$, called the generator of $T(\cdot)$, such that $(w, \infty) \subset$ $\rho(A)$ and $(\lambda-A)^{-1} x=\int_{0}^{\infty} \lambda^{n} e^{-\lambda t} T(t) x d t$ for all $x \in X$ and $\lambda>w$. For the definitions and basic properties we refer to Arendt [1], and Tanaka and Miyadera [11].

It is known [1, Proposition 3.3] that $\int_{0}^{t} T(s) x d s \in D(A)$ and $A \int_{0}^{t} T(s) x d s=$ $T(t) x-\left(t^{n} / n !\right) x$ for all $x \in X ; \int_{0}^{t} T(t) A x d s=T(t) x-\left(t^{n} / n !\right) x$ for all $x \in D(A)$. Hence, if we put $A_{t}:=(n+1) ! t^{-n-1} \int_{0}^{t} T(s) d s$ and $B_{t}:=-(n+1) ! t^{-n-1} \int_{0}^{t} \int_{0}^{s} T(u) d u d s$ for $t>0$, then the closedness of $A$ implies that $B_{t} A \subset A B_{t}=I-A_{t}$ and $A_{t} A \subset$ $A A_{t}=(n+1) ! t^{-n-1} T(t)-(n+1) t^{-1} I$. Thus (c) holds if $t^{-n-1} T(t)$ converges strongly to 0 as $t \rightarrow \infty$. In particular, both (a) and (c) will hold in case $\|T(t)\|=O\left(t^{n}\right)(t \rightarrow \infty)$. To verify (d) let $x^{*} \in R(A)^{\perp}$. Then $\left\langle T(u) x-\left(u^{n} / n !\right) x, x^{*}\right\rangle=\left\langle A \int_{0}^{u} T(s) x d s, x^{*}\right\rangle$ $=0$ for all $u \geqq 0$, so that

$$
\begin{aligned}
\left\langle x, B_{t}^{*} x^{*}\right\rangle & \left.=\left\langle B_{t} x, x^{*}\right\rangle=-(n+1) ! t^{-n-1} \int_{0}^{t} \int_{0}^{s} T(u) x, x^{*}\right\rangle d u d s \\
& =-(n+1) t^{-n-1} \int_{0}^{t} \int_{0}^{s} u^{n} d u d s\left\langle x, x^{*}\right\rangle
\end{aligned}
$$




$$
=-\frac{t}{n+2}\left\langle x, x^{*}\right\rangle \quad \text { for all } \quad x \in X
$$

That is, the condition (d) holds with $\phi(t)=-\frac{t}{n+2}$.

On the other hand, if $\left\|A_{t}\right\| \leqq M$ for all $t \geqq 0$, then

$$
\begin{aligned}
\left\|(\lambda-A)^{-1} x\right\| & \leqq\left\|\int_{0}^{\infty} e^{-\lambda t} \lambda^{n} T(t) x d t\right\| \\
& \leqq \lambda^{n+1} \int_{0}^{\infty} e^{-\lambda t}\left\|\int_{0}^{t} T(s) x d s\right\| d t \\
& \leqq \frac{\lambda^{n+1}}{(n+1) !} M \int_{0}^{\infty} e^{-\lambda t} t^{n+1} d t\|x\| \\
& =\frac{M}{\lambda}\|x\|
\end{aligned}
$$

for all $x \in X$ and $\lambda>0$, so that $\left\{\lambda(\lambda-A)^{-1}\right\}_{\lambda>0}$ is a system of almost invariant integrals and $\left\{(\lambda-A)^{-1}\right\}_{\lambda>0}$ the associated system of companion integrals (see [8, Example V]).

Now Theorems 1 and 2 can be applied to the two pairs $\left\{\left\{A_{t}\right\},\left\{B_{t}\right\}\right\}$ and $\left\{\left\{\lambda(\lambda-A)^{-1}\right\},\left\{(\lambda-A)^{-1}\right\}\right\}$ to deliver the next theorem, which is concerned with the equivalence of the following conditions:

(S1) $y \in R(A)$;

(S2) $\sup _{\lambda>0}\left\|(\lambda-A)^{-1} y\right\|<\infty$;

(S3) $x=\mathrm{w}-\lim _{k \rightarrow \infty}\left(A-\lambda_{k}\right)^{-1} y$ exists for some sequence $\left\{\lambda_{k}\right\} \rightarrow 0^{+}$;

(S4) $x=\mathrm{s}-\lim _{\lambda \rightarrow 0^{+}}(A-\lambda)^{-1} y$ exists ;

(S5) $\sup _{t>0}\left\|t^{-n-1} \int_{0}^{t} \int_{0}^{s} T(u) y d u d s\right\|<\infty$;

(S6) $x=-\mathrm{W}-\lim _{k \rightarrow \infty}(n+1) ! t_{k}^{-n-1} \int_{0}^{t_{k}} \int_{0}^{s} T(u) y d u d s$ exists for some sequence $\left\{t_{k}\right\} \rightarrow \infty$

(S7) $\quad x=-\mathrm{s}-\lim _{t \rightarrow \infty}(n+1) ! t^{-n-1} \int_{0}^{t} \int_{0}^{s} T(u) d u d s$ exists ;

(S8) $\sup _{t>0}\left\|t^{-n} \int_{0}^{t} T(s) y d s\right\|<\infty$.

Theorem 4. Let $T(\cdot)$ be a non-degenerate, exponentially bounded, n-times integrated semigroup on $X$, and $A$ be its generator. Suppose that $\left\|(n+1) ! t^{-n-1} \int_{0}^{t} T(s) x d s\right\| \leqq M\|x\|$ for all $x \in X$ and $t>0$ and that $t^{-n-1} T(t) \rightarrow 0$ 
strongly as $t \rightarrow \infty$.

(i) If $X$ is reflexive, then the conditions (S1)-(S7) are equivalent to each other.

(ii) If $X=L_{1}(S, \Sigma, \mu)$ with $\mu$ a $\sigma$-finite measure, and if $M=1$, then conditions (S1), (S2), and (S5) are equivalent; they are also equivalent to (S3), (S4), (S6) and (S7) in case $\mu$ is a finite measure and $\left\|t^{-n-1} \int_{0}^{t} T(s) f d s\right\|_{\infty} \leqq K\|f\|_{\infty}$ for all $f \in$ $L_{\infty}(S, \Sigma, \mu)$ and all $t>0$.

Remark. If $T(\cdot)$ satisfies the growth condition $\|T(t)\| \leqq M t^{n} /(n+1) !, t \geqq 0$, then the hypothesis of Theorem 4 is satisfied and (S8) can be added as another equivalent condition. In fact, it is easy to see that (S1) $\Rightarrow(\mathrm{S} 8) \Rightarrow(\mathrm{S} 5)$ in this case.

The following corollary for contraction $C_{0}$-semigroups on $L_{1}(S, \Sigma, \mu)$ is a specialization of Theorem 4; the first part of it is due to Krengel and Lin [6] (see also [9]).

Corollary 5. Let $A$ be the generator of a $C_{0}$-semigroup $T(\cdot)$ of contractions on $L_{1}(S, \Sigma, \mu)$, with $\mu$ a $\sigma$-finite measure. Then with $n=0$ the conditions (S1), (S2), (S5), and (S8) are equivalent. If, in addition, $\mu$ is finite and $\sup _{t>0}\left\|t^{-1} \int_{0}^{t} T(s) f d s\right\|_{\infty} \leqq K\|f\|_{\infty}$ for all $f \in L_{\infty}(S, \Sigma, \mu)$, then (S1)-(S8), with $n=0$, all are equivalent.

For a given function $f \in R(A)$ in $L_{p}(S, \Sigma, \mu), 1 \leqq p<\infty$, we now consider the almost everywhere convergence of $B_{t} f$. Suppose a pointwise ergodic theorem for the system $\left\{A_{t}\right\}$ holds so that $A_{t} x$ converges almost everywhere on $S$ for all $x \in L_{p}$. Then for any solution $g$ of the equation $A g=f, B_{t} f=$ $B_{t} A g=\left(I-A_{t}\right) g$ surely converges almost everywhere. If $A g=f$ has a solution $g$ in $D(A) \cap \overline{R(A)}$ (This is always the case when $\left\{A_{t}\right\}$ is mean ergodic, i.e. $D(P)=X)$, then $A_{t} g$ converges to $P g=0$ almost everywhere on $S$ and $B_{t} f$ converges to $g$ almost everywhere on $S$. In what follows we deduce from Theorem 4, Corollary 5 , and the Cesàro and Abelian pointwise ergodic theorems in [5] a pointwise convergence theorem for the approximate solutions $\left\{B_{t} f\right\}$ of $A g=f$.

Let $A$ be the generator of a $C_{0}$-semigroup $T(\cdot)$ of contractions on $L_{1}(S, \Sigma, \mu)$ such that, for some $K \geqq 1, \sup _{t>0}\|T(t) f\|_{\infty} \leqq K\|f\|_{\infty}$ for all $f \in L_{1}(S, \Sigma, \mu) \cap$ $L_{\infty}(S, \Sigma, \mu)$. Then, given any $p \in[1, \infty)$, each $T(t)$ can be extended to a linear operator, still denoted by $T(t)$, on $L_{p}(S, \Sigma, \mu)$ with $\|T(t)\|_{p} \leqq K$ and $\{T(t) ; t \geqq 0\}$ is also a $C_{0}$-semigroup of operators on $L_{p}(S, \Sigma, \mu)$ (cf. $[5$, p. 96]). Let $A$ still denote the generator of the semigroup thus obtained. Under these assumptions we can formulate the following Theorem. 
Theorem 6. Let $1 \leqq p<\infty$. (i) $f \in L_{p}(S, \Sigma, \mu)$ satisfies $\sup _{t>0}\left\|\int_{0}^{t} T(u) f d u\right\|_{p}<\infty$ if and only if $A g=f$ is solvable in $L_{p}(S, \Sigma, \mu)$. (ii) If $A g=f$ is solvable, then the limits

$$
\lim _{t \rightarrow \infty} t^{-1} \int_{0}^{t} \int_{0}^{u}(-T(v) f)(s) d v d u \text { and } \lim _{\lambda \rightarrow 0^{+}}\left[(A-\lambda)^{-1} f\right](s)
$$

exist and coincide almost everywhere on $S$. (iii) If $1<p<\infty$,or if $p=1$ and $\mu$ is a finite measure, then the limits in (ii) converge in $\|\cdot\|_{p}$ and the limit function $g$ is the unique solution of $A g=f$ in the $\|\cdot\|_{p}$-closure of $R\left(A \mid L_{p}\right)$.

We end this section with a concrete application to the equation $\Delta g=f$ in $L_{p}\left(R^{n}\right), 1 \leqq p<\infty$, where $\Delta$ is the Laplacian $\sum_{i=1}^{n} \frac{\partial^{2}}{\partial x_{i}^{2}}$. It is known that $\Delta$ generates the Gauss-Weierstrass semigroup $T(\cdot)$, which is defined by $T(0)=I$ and

$$
(T(t) f)(x)=(4 \pi t)^{-n / 2} \int_{R^{n}} \exp \left(-\frac{\mid x-y !^{2}}{4 t}\right) f(y) d y, \quad f \in L_{p}\left(R^{n}\right), t>0 .
$$

This is a $C_{0}$-semigroup of contractions on $L_{p}\left(R^{n}\right)$. Hence we can formulate the following specialization of Theorem 6 .

Corollary 7. Let $f$ be a function in $L_{p}\left(R^{n}\right), 1 \leqq p<\infty$. Then the equation $\Delta g=f$ is solvable if and only if

$$
\sup _{\lambda>0}\left\|\int_{0}^{\infty} e^{-\lambda t}(4 \pi t)^{-n / 2} \int_{R^{n}} \exp \left(-\frac{|x-y|^{2}}{4 t}\right) f(y) d y d t\right\|_{p}<\infty,
$$

if and only if

$$
\sup _{t>0}\left\|\int_{0}^{t}(4 \pi s)^{-n / 2} \int_{R^{n}} \exp \left(-\frac{|x-y|^{2}}{4 s}\right) f(y) d y d s\right\|_{p}<\infty .
$$

When $p>1$, a solution is given by

$$
\begin{aligned}
g(x) & =-\lim _{\lambda \rightarrow 0^{+}} \int_{0}^{\infty} e^{-\lambda t}(4 \pi t)^{-n / 2} \int_{R^{n}} \exp \left(-\frac{|x-y|^{2}}{4 t}\right) f(y) d y d t, \\
& =-\lim _{t \rightarrow \infty^{+}} t^{-1} \int_{0}^{t} \int_{0}^{s}(4 \pi u)^{-n / 2} \int_{R^{n}} \exp \left(-\frac{|x-y|^{2}}{4 u}\right) f(y) d y d u d s,
\end{aligned}
$$

the convergence being valid in the sense of pointwise almost cverywhere as well as in the sense of $\|\cdot\|_{p}$.

\section{$\S 4$. Generators of Cosine Operator Functions}

A strongly continuous family $\{C(t) ; t \in R\}$ of bounded linear operators on $X$ is called a cosine operator function if $C(0)=I$ and $C(t+s)+C(t-s)=2 C(t) C(s)$, 
$s, t \in R$. The associated sine function $S(\cdot)$ is defined by $S(t) x=\int_{0}^{t} C(s) x d s$, $x \subseteq X$. The generator $A:=C^{\prime \prime}(0)$ is a densely defined closed operator. There exist $M \geqq 1$ and $w \geqq 0$ such that $\|C(t)\| \leqq M e^{w|t|}, t \in R$. The resolvent set $\rho(A)$ contains all $\lambda^{2}$ with $\lambda>w$, and for each such $\lambda$

$$
\lambda\left(\lambda^{2}-A\right)^{-1} x=\int_{0}^{\infty} e^{-\lambda t} C(t) x d t \quad \text { for all } \quad x \in X .
$$

See, e.g., Sova $[10]$ for these and other properties of $C(\cdot)$.

For $t>0$, let $A_{t}:=2 t^{-2} \int_{0}^{t} S(s) d s$ and $B_{t}:=-2 t^{-2} \int_{0}^{t} \int_{0}^{s} \int_{0}^{u} S(v) d v d u d s$. Then we have $B_{t} A \subset A B_{t}=I-A_{t}, A_{t} A \subset A A_{t}=2 t^{-2}(C(t)-I)$, and $B_{t}^{*} x^{*}=\frac{t^{2}}{12} x^{*}$ for all $x^{*} \in N\left(A^{*}\right)$ (see [8], Example VII). Hence $\left\{A_{t}\right\}$ is a system of almost invariant integrals for $A+I$ and $\left\{B_{t}\right\}$ is its associated system of companion integrals if $\left\|A_{t}\right\| \leqq M$ and if $t^{-2} C(t) \rightarrow 0$ strongly as $t \rightarrow \infty$. Moreover, as was in the case of semigroup, the condition $\left\|A_{t}\right\| \leqq M$ also implies that $\left\{\lambda(\lambda-A)^{-1}\right\}_{\lambda>0}$ is a system of almost invariant integrals and $\left\{(\lambda-A)^{-1}\right\}_{\lambda>0}$ the associated system of companion integrals.

From Theorems 1 and 2 we can immediately deduce the next theorem, which is concerned with the equivalence among the following conditions:

(C1) $y \in R(A)$;

(C2) $\sup _{\lambda>0}\left\|(\lambda-A)^{-1} y\right\|<\infty$;

(C3) $x=\mathrm{w}-\lim _{k \rightarrow \infty}\left(A-\lambda_{k}\right)^{-1} y$ exists for some sequence $\left\{\lambda_{k}\right\} \rightarrow 0^{+}$;

(C4) $x=\mathrm{s}-\lim _{\lambda \rightarrow 0^{+}}(A-\lambda)^{-1} y$ exists ;

(C5) $\sup _{t>0}\left\|t^{-2} \int_{0}^{t} \int_{0}^{s} \int_{0}^{u} S(v) y d v d u d s\right\|<\infty$;

(C6) $x=-\mathrm{w}-\lim _{k \rightarrow \infty} 2 t_{k}^{-2} \int_{0}^{t_{k}} \int_{0}^{s} \int_{0}^{u} S(v) y d v d u d s$ exists for some sequence $\left\{t_{k}\right\} \rightarrow \infty$;

(C7) $x=-\mathrm{s}-\lim _{t \rightarrow \infty} 2 t^{-2} \int_{0}^{t} \int_{0}^{s} \int_{0}^{u} S(v) y d v d u d s$ exists ;

(C8) $\sup _{t>0}\left\|\int_{0}^{t} S(s) y d s\right\| \leqq \infty$.

Theorem 8. Let $C(\cdot)$ be a cosine operator function on $X$. Suppose that $\left\|2 t^{-2} \int_{0}^{t} S(s) d s\right\| \leqq M$ for all $t>0$ and $t^{-2} C(t) \rightarrow 0$ strongly as $t \rightarrow \infty$.

(i) If $X$ is reflexive, then condition (C1)-(C7) are equivalent to each other.

(ii) If $X=L_{1}(S, \Sigma, \mu)$ with $\mu a \sigma$-finite measure, and if $M=1$, then conditions (C1), (C2), and (C5) are equivalent; moreover, they are also equivalent to condi- 
tions (C3), (C4), (C6), and (C7) when $\mu$ is finite and $\left\|2 t^{-2} \int_{0}^{t} S(s) f d s\right\|_{\infty} \leqq K\|f\|_{\infty}$ for all $f \in L_{\infty}(S, \Sigma, \mu)$.

Remark. If $\|C(t)\| \leqq M$ for all $t \geqq 0$, then both cases (i) and (ii), the condition (C8) can be added as an equivalent condition, because $(\mathrm{C} 1) \Rightarrow(\mathrm{C} 8) \Rightarrow(\mathrm{C} 2)$.

\section{References}

[1] Arendt, W., Vector valued Laplace transforms and Cauchy problems, Israel J. Math., 59 (1987), 327-352.

[2] Dotson, W.G. Jr., An application of ergodic theory to the solution of linear functional equations in Banach spaces, Bull. Amer. Math. Sec., 75 (1969), 347-352.

[3] Dunford, N. and Schwartz, J. T., Linear Operators, Interscience, New York, 1958.

[4] Eberlein, W.F., Abstract ergodic theorems and weak almost periodic functions, Trans. Amer. Math. Soc., 67 (1949), 217-240.

[5] Hasegawa, S., Sato, R. and Tsurumi, S., Vector valued ergodic theorems for a one-parameter semigroup of linear operators, Tôhoku Math. J., 30 (1978), 95-106.

[6] Krengel, U. and Lin, M., On the range of the generator of a Markovian semigroup, Math. Z., 185 (1984), 553-565.

[7] Lin, M. and Sine, R., Ergodic theory and the functional equation $(I-T) x=y, J$. Operator Theory, 10 (1983), 153-166.

[8] Shaw, S.-Y., Mean ergodic theorems and linear functional equations, J. Funct. Anal., 87 (1989), 428-441.

[9] - On the range of a closed operator, J. Operator Theory, 22 (1989), 157163.

[10] Sova, M., Cosine operator functions, Rozprawy Math., 49 (1966), 1-47.

[11] Tanaka, N. and Miyadera, I., Some remarks on $C$-semigroups and integrated semigroups, Proc. Japan Acad., 63A (1987), 139-142.

[12] Yosida, K. and Hewitt, E., Finitely additive measures, Trans. Amer. Math. Soc., 72 (1952), 46-66. 
